\title{
INSTRUMENT DEVELOPMENT FOR ACETABULAR ROOF PLASTY IN CASE OF DYSPLASTIC ACETABULAR REPLACEMENT
}

\author{
János Szabó, Sándor Manó, Ádám Lőrincz, Gyula Győrfi, László Kiss \\ Department of Orthopaedic Surgery, Medical and Health Science Center, University \\ of Debrecen \\ szabojan@med.unideb.hu
}

\begin{abstract}
The authors report on the development of special surgical instruments required for the new Intraosseal Structural Graft (ISG) technique in case of dysplastic acetabular replacement presently under introduction at our Institute. One of novelties of procedure is the development of a proximally hinged cortico-spongious plate in the cranial quadrant of the acetabulum that is opened laterally.

The instruments were developed, tested, and modified based on experiments on 14 3D models and 19 cadaver hips. For the cortico-spongious plate a 1 and 3 step method was developed and the prototypes of the chisels required were made. The open the bone block laterally a threaded device was planned and developed.

Based on the experiments the 1 step method was discarded, because the device used for opening the bone block laterally proved difficult, and so it was not further developed.

The instruments required for the 3 step method was finalized and since than 2 successful operations were performed with them.

With the aid of these special surgical instruments the ISG technique is easier, quicker and safer to perform.
\end{abstract}

Keywords: hip dysplasia, prosthesis, graft, special chisels

\section{Introduction}

The replacement of the dysplatic acetabulum is a surgically demanding procedure. One of the most important aspects is the reconstruction of the supero-lateral defect of the acetabulum. ${ }^{1-5}$

During the development of the Intraosseal Structural Graft (ISG) technique our research group also worked on the surgical instruments required during the operation. During the procedure in the cranial quadrant of the acetabulum a proximally hinged cortico-spongious plate is fashioned that is opened laterally and a wedge shaped graft made from the femoral head is impacted. This is stabilized by 1-2 cortical screws with washers (Figure 1).

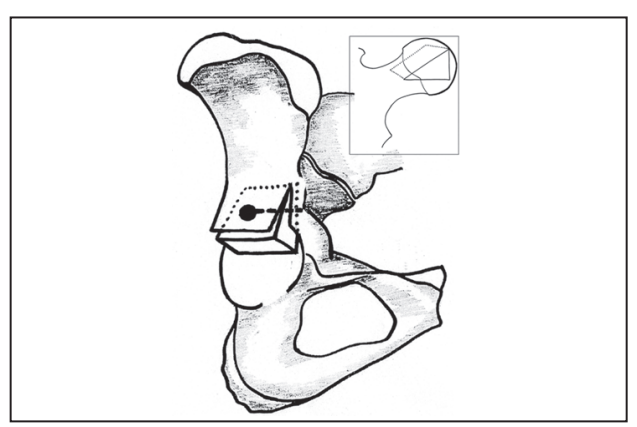

Figure 1. Schematic drawing of the ISG technique 


\section{Methods and materials}

In the ISG technique there are two surgical steps which can be made easier if special instruments are at our disposal. During the development of the instruments it was a basic requirement that they should be simple, easy to use, and economical.

\section{The preparation} of the cortico-spongious block

Prior to development morphological examinations were performed on 3D models in order to determine the parameters of the instruments. It can be seen on the cranial to caudal sections in the supra-acetabular region that the iliac crest thins towards the cranial direc- tion. This is most prominent at dorsal plane of the acetabulum (Figure 2 white arrows).

Following this we measured the thickness of the pelvic wall in 4 places in the supra-acetabular region where the proximal hinged corticospongious plate is planned to be made (Figure 3). The plate is calculated to be $4 \times 3 \mathrm{~cm}$. The thickness measurements were made at the rim of the acetabulum and $4 \mathrm{~cm}$ cranial to it.

Fourteen pelvic models were used that cover the most frequently sizes occurring during hip replacement surgery (Table 1). It can be seen that the thinnest part of the pelvis in this area is the proximal-dorsal point. The lowest value was $10 \mathrm{~mm}$, which means that the bone block at this point cannot be thicker than this.
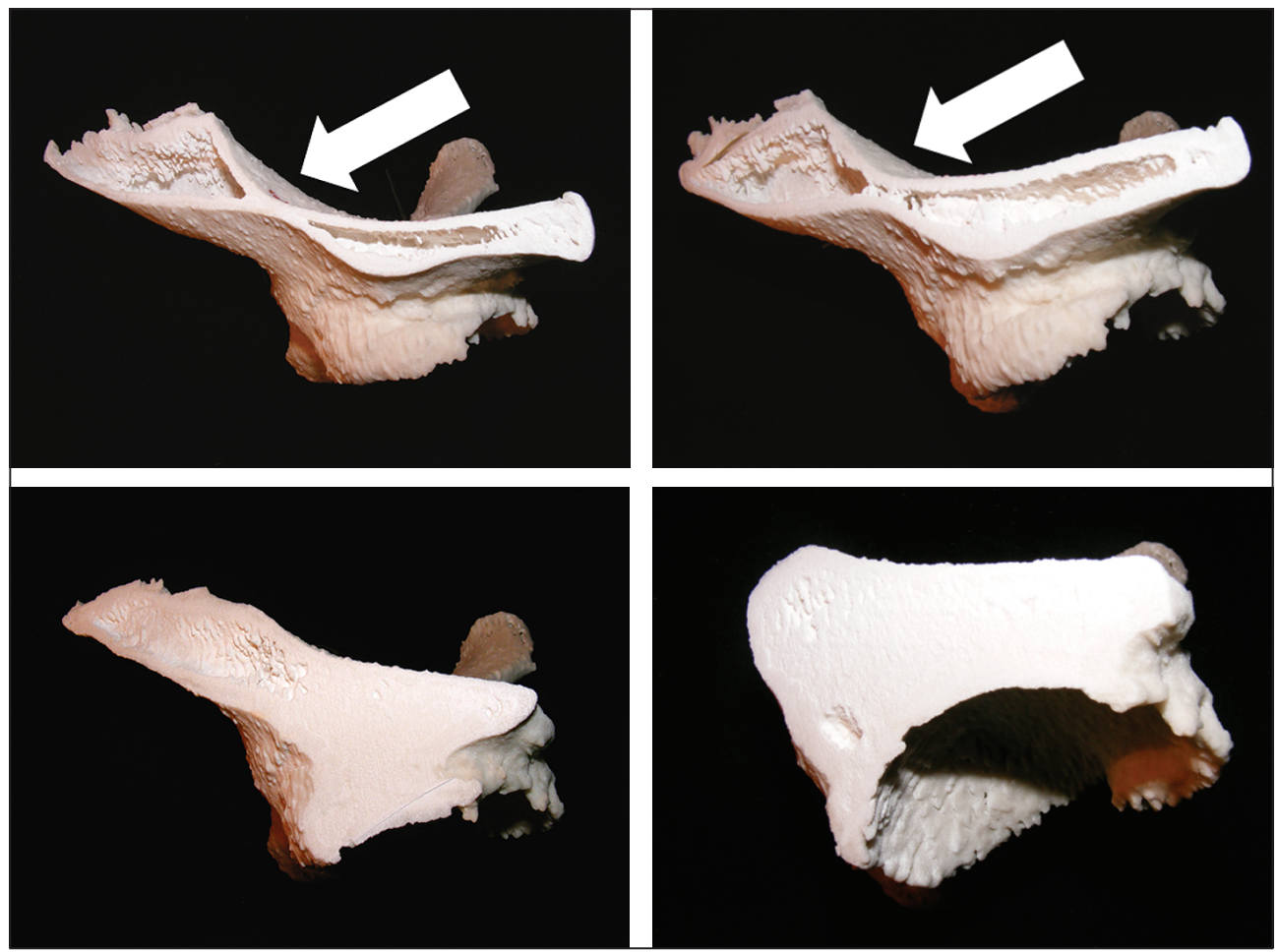

Figure 2. The supra-acetabular region on the horizontal sections of the right hip.

The white arrows show the thinning of the pelvis 


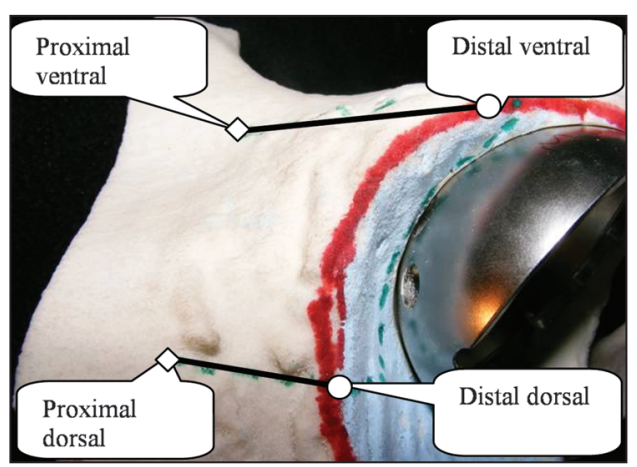

Figure 3. The points of thickness measurements

\begin{tabular}{|r|c|c|c|c|c|}
\hline \multirow{2}{*}{ No. } & \multirow{2}{*}{ Cup } & \multicolumn{2}{|c|}{ Distal } & \multicolumn{2}{c|}{ Proximal } \\
\cline { 3 - 6 } & & ventral & dorsal & ventral & dorsal \\
\hline 1 & 46 & 19 & 31 & 16 & 11 \\
\hline 2 & 48 & 19 & 33 & 13 & 12 \\
\hline 3 & 48 & 15 & 37 & 15 & 13 \\
\hline 4 & 50 & 22 & 45 & 21 & 14 \\
\hline 5 & 50 & 13 & 26 & 14 & 13 \\
\hline 6 & 50 & 23 & 47 & 19 & 15 \\
\hline 7 & 52 & 15 & 40 & 17 & 10 \\
\hline 8 & 52 & 23 & 47 & 19 & 15 \\
\hline 9 & 54 & 22 & 25 & 19 & 10 \\
\hline 10 & 54 & 16 & 34 & 17 & 17 \\
\hline 11 & 54 & 17 & 30 & 15 & 21 \\
\hline 12 & 54 & 22 & 43 & 15 & 11 \\
\hline 13 & 58 & 20 & 33 & 17 & 15 \\
\hline 14 & 60 & 24 & 38 & 11 & 19 \\
\hline
\end{tabular}

Table 1. The thickness of the bone in the four measured points in relation to the size of the acetabular cup (mm)
Two ideas were examined for the instrument development. Both were tested on models and cadaver experimentations. During the development great emphasis was taken to protect the gluteal muscles and to avoid pelvic perforation.

\section{1. a) One step concept}

The U shaped chisel would make it possible to develop the cortico-spongious block in one step (Figure 4).

\section{The one step concept}

Benefits:

- quick;

- simple, no need to reconcile each step;

- less soft tissue damage.

Disadvantages:

- hard to control, therefore the probability of pelvic perforation increases;

- due to the length of the chisel great force is needed;

- because of different sized acetabula a series is required.

\section{1.b) Three step concept}

Two chisels are required. The $\mathrm{T}$ shaped raspchisel (Figure 5) is used for the frontal cut while the flat rasp-chisel (Figure 6) is used to make the horizontal osteotomy.
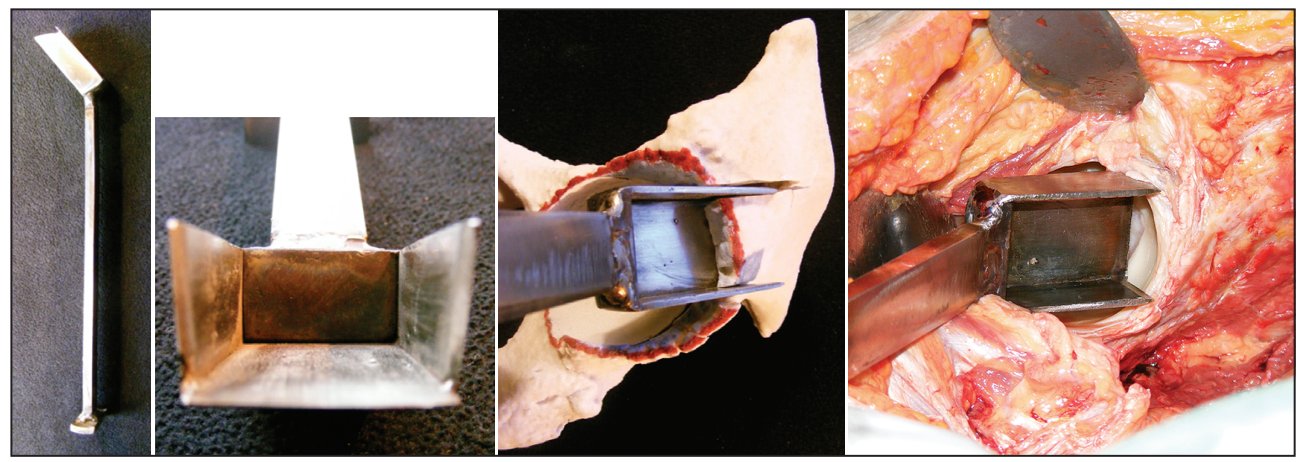

Figure 4. The $\mathrm{U}$ shaped chisel and its use on a model and in cadaver examinations 


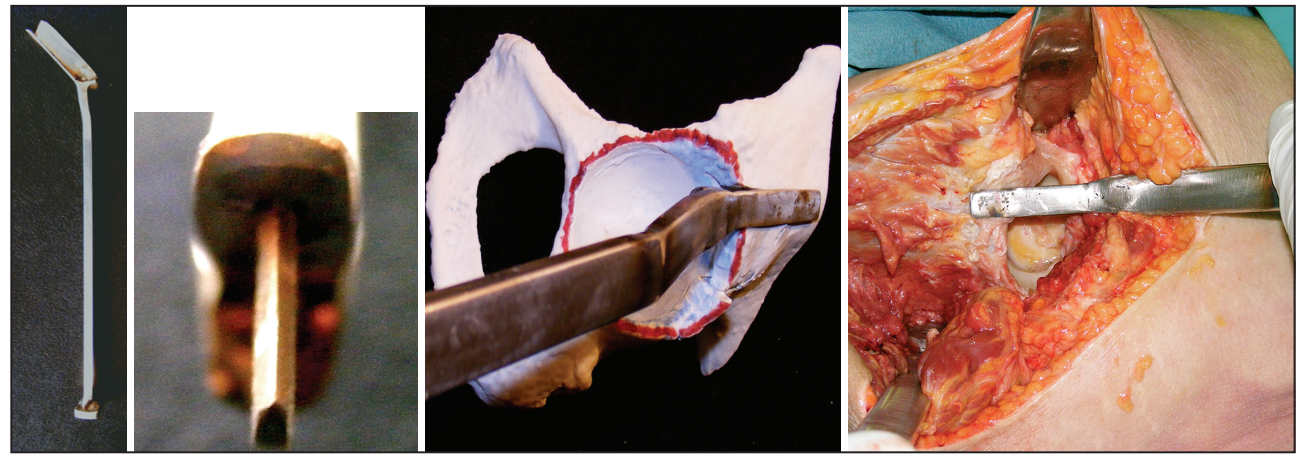

Figure 5. The $\mathrm{T}$ shaped rasp-chisel and its use on a model and in cadaver examinations
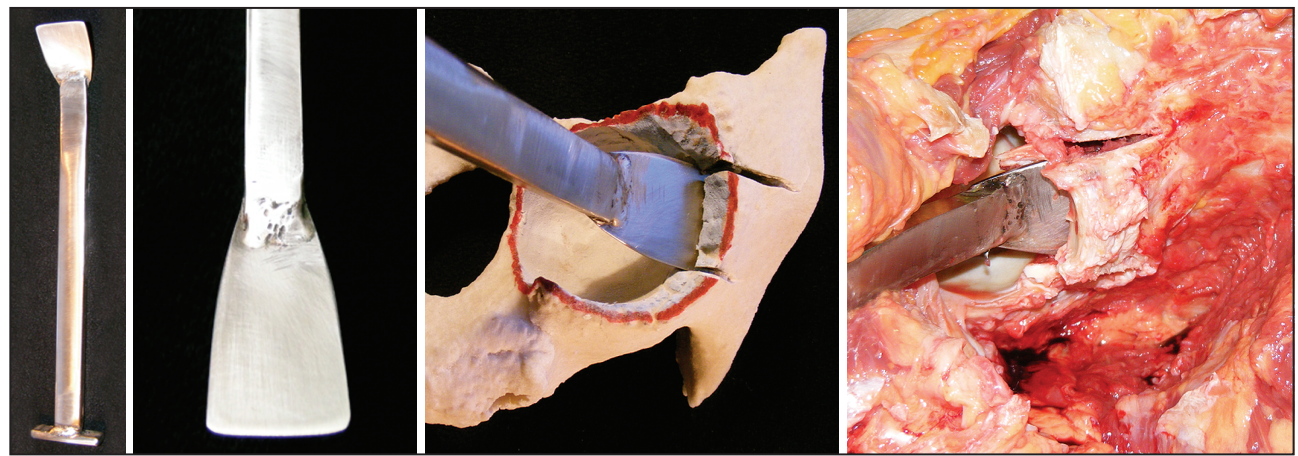

Figure 6. The flat rasp-chisel and its use on a model and in cadaver examinations

The horizontal blade of the $\mathrm{T}$ instrument guides the chisel on the exterior cortical of the pelvis. This way the detachment of the gluteal muscles is less traumatic, and by choosing the proper width of the vertical blade pelvic perforation can be avoided. Based on model examinations taking into consideration the periosteal coverage the width of the vertical blade was chosen to be $10 \mathrm{~mm}$. The length of the cranial direction chiseling is $30 \mathrm{~mm}$ in case the implanted acetabular cup is under $50 \mathrm{~mm}$ and $40 \mathrm{~mm}$ if it is larger.

For the sagittal plane chiseling we developed the instrument shown in Figure 6. The tip of the chisel is asymmetric this directs the head of the instrument towards the external cortical bone. In this manner it decreases the risk of pelvic perforation.
The three step concept

Benefits:

- the shape and size of the graft is adaptable;

- the individual steps require less force;

- the work is easier to control.

Disadvantages:

- slower, the individual steps require more planning;

- greater soft tissue damage;

- the instruments because of left and right versions are more expensive.

\section{Modifications}

The basic concept was to design the instruments based on available models and to test these prototypes on similar models and cadavers. Since all the work phases were performed 


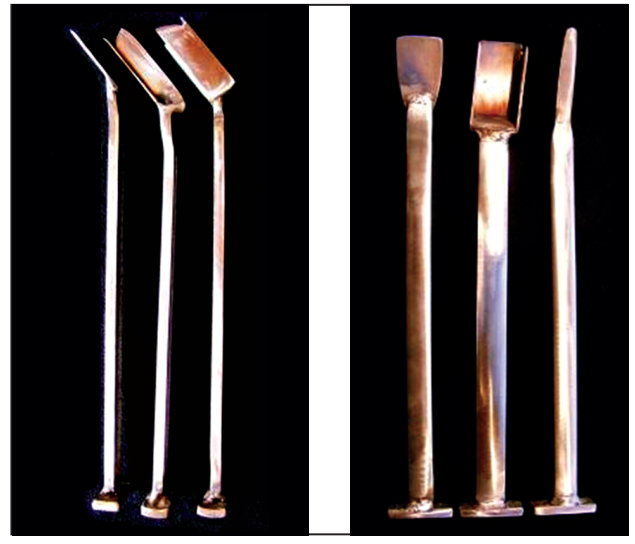

Figure 7. The first prototypes

by our team - including the prototype manufacturing - we had the chance to modify or discard every concept. Figure 7 shows the first prototype series.
During the cadaver experimentation using the final prototypes we realized that the 45 degree angle between the shaft and the head of the chisel although allows good reach of the supraacetabular region; this also decreases the amount of force that can be applied. To solve this problem we modified the instruments by making the distal third bayonet shaped with a 30-30 degrees break (Figure 8-9).

The modifications solved the problems with the applied force, but new problems arose. The blade of the $\mathrm{U}$ chisel was so long that even after modification large force was required to pass through the subchondral bone. Also due to its size and shape it was difficult to maneuver the instrument in the acetabulum, and the cortico-spongious block frequently became entrapped into the head of the chisel and dur-

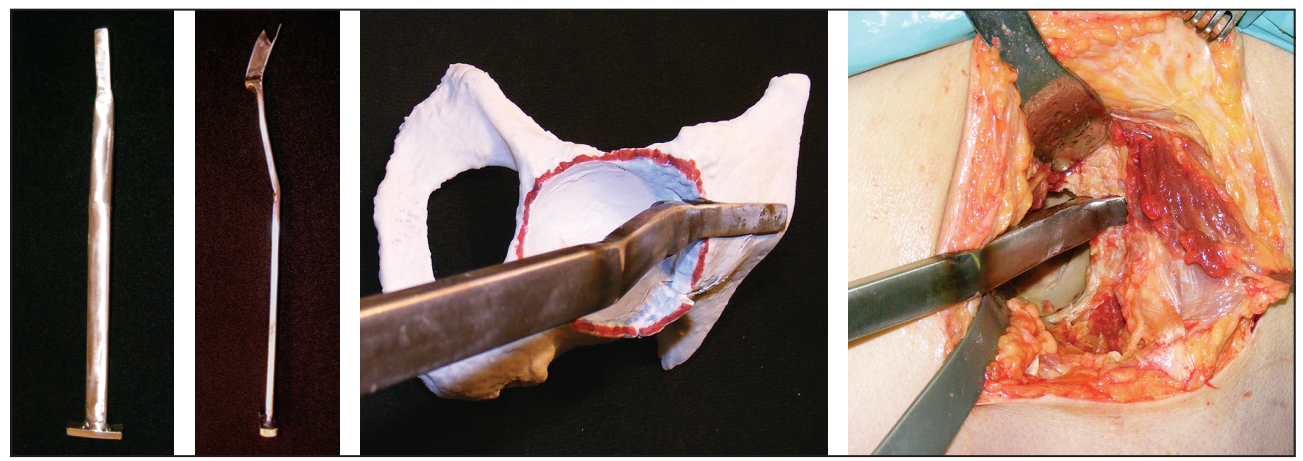

Figure 8. The modified T chisel
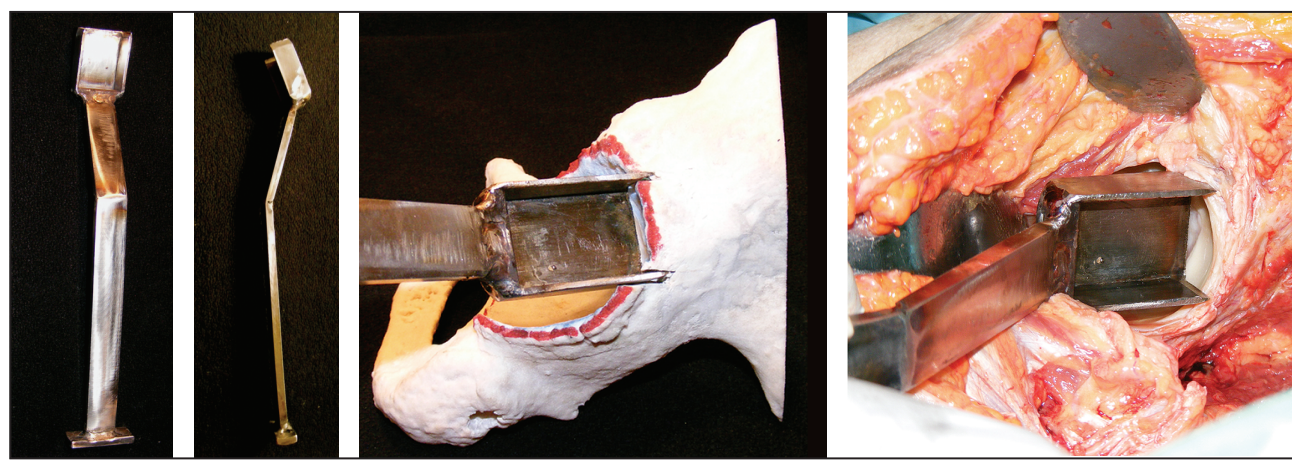

Figure 9. The modified U chisel 


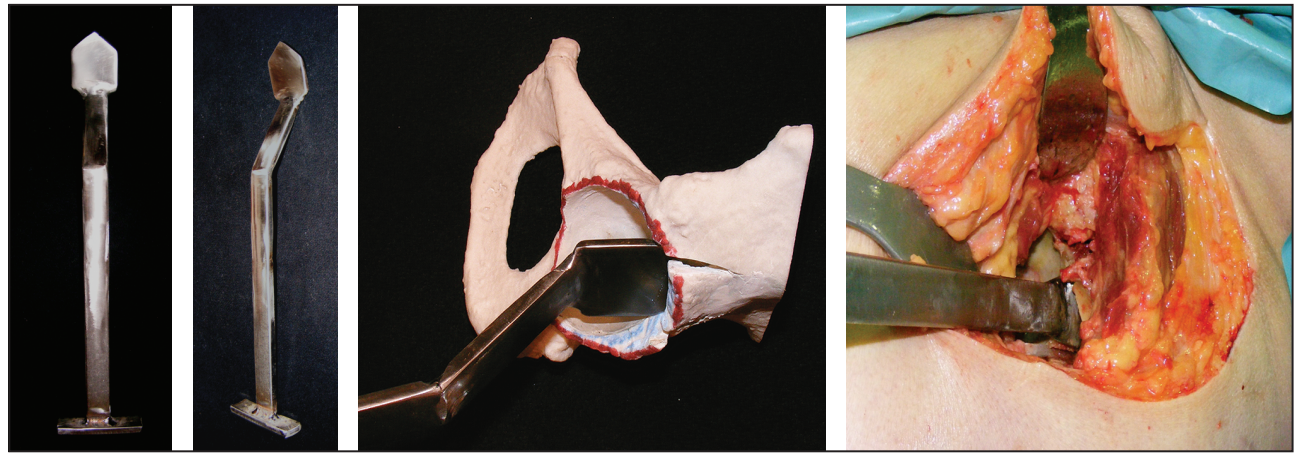

Figure 10. The final prototype version of the flat chisel
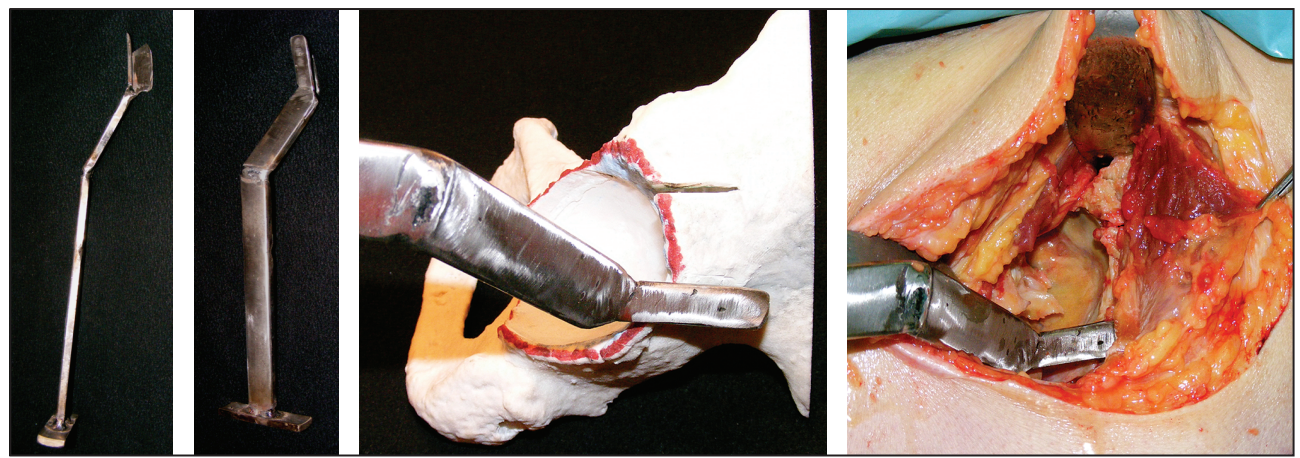

Figure 11. The final prototype version of the $\mathrm{T}$ shaped chisel

ing removal it broke the proximal hinge. Examining these results we discarded the one step concept.

During the modification of the flat chisel the head was also modified. The new sharp design makes it easier to begin and control the osteotomy (Figure 10). Though the asymmetric sharp end, due to good experience was maintained.

The bayonet redesign of the $\mathrm{T}$ instrument besides increasing the amount of force that can be applied also made the ventral approach easier. Dorsally there was serious difficulty with the proximal femur. This problem was solved by bending the shaft of the chisel 30-30 degrees in the frontal and saggital plane (Figure 11). It can be seen from the figure that this modification allows for better aiming in the dorsal proximal direction. Unfortunately this requires a left and a right version of the instrument.

Based on the final and tested prototype plans were drawn up as can be seen in Figure 12 and the final version of the chisels was manufactured by a company (Figure 13).

\section{The lateral bending of the cortico-spongious block}

A combined instrument was made to ease the lateral bending of the block (Figure 14).

The gripping device rests on the acetabulum. The blade at the end of a threaded rod is inserted into the osteotomy. By lifting the han- 


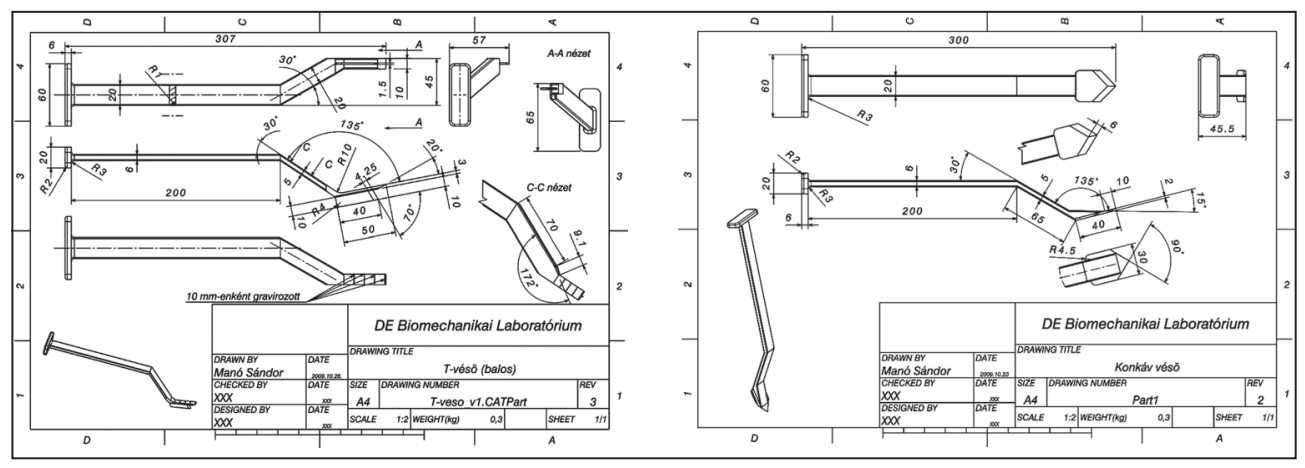

Figure 12. Plans of the finalized version

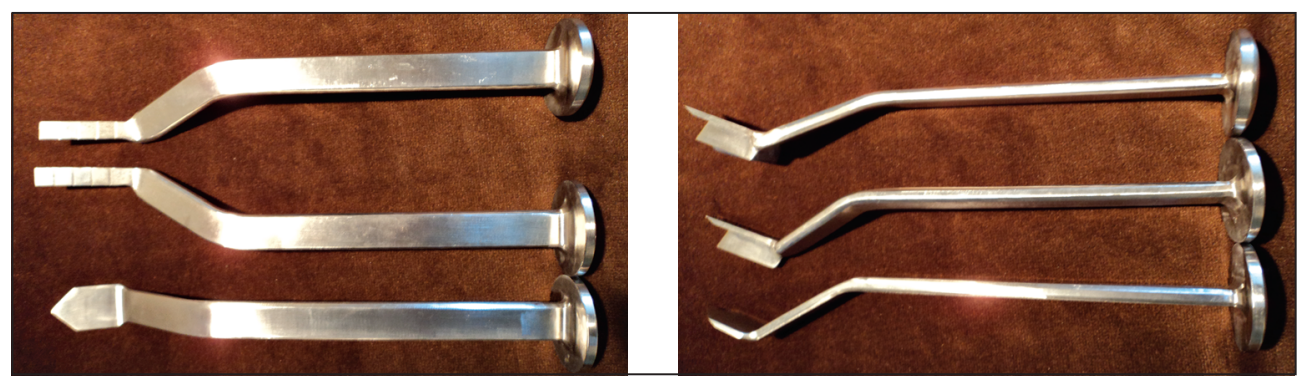

Figure 13. The final version of the instruments

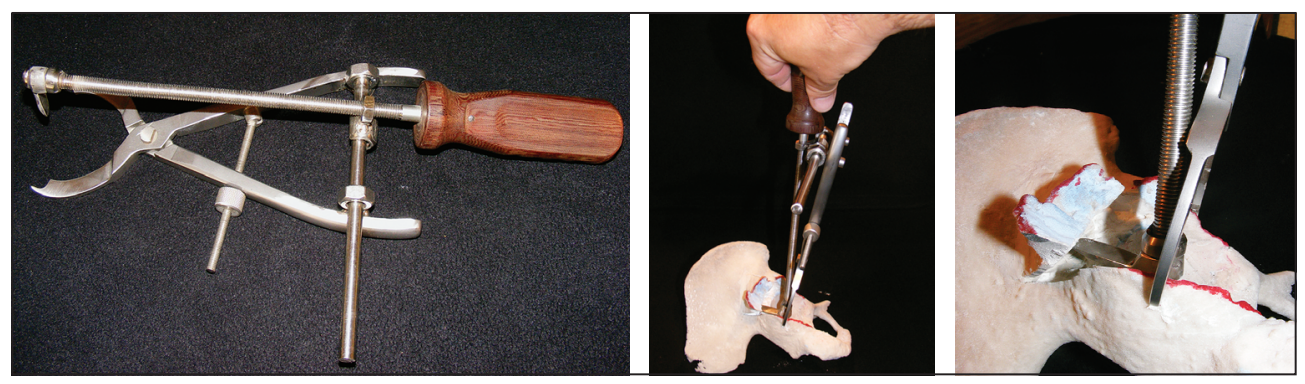

Figure 14. The use of the instrument required for the lateral bending of the block on a model

dle a lateral displacement of the cortico-spongious block occurs. The advantage of the device is that due the threaded nature the procedure can be carried out gently and under good control. Since the grip is applied to the edge of the acetabulum during lateral displacement the fracture of the internal cortices can be avoided. By calibrating the shaft this device can also be used for measurement purposes.
The prototype was encouraging during the model tests, but in the cadaver examination it proved to be difficult to use and uncontrollable so we gave up on further development.

Numerous other instruments were tried, but the flat chisel proved to be the most simplistic and reliable. Since the instrument has a relatively wide head there is no need to fear internal cortical fracture. 

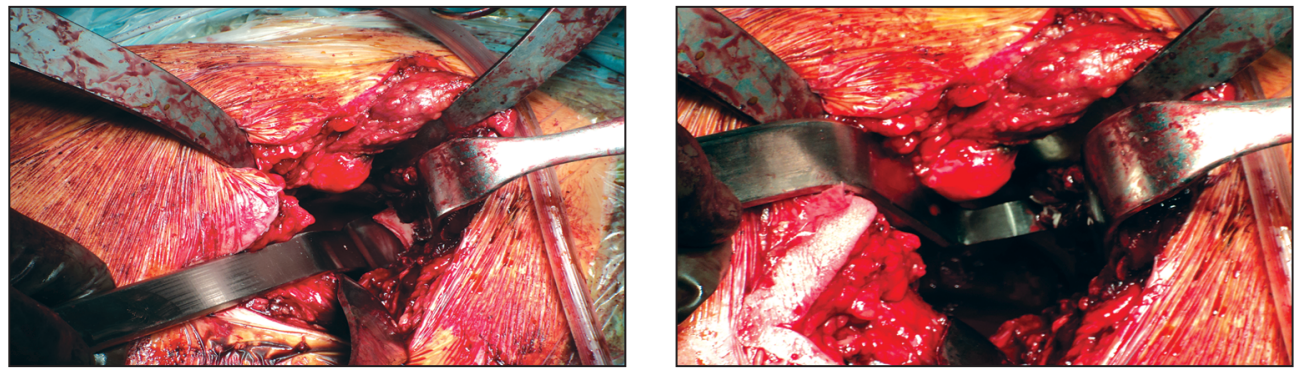

Figure 15. Intraoperative use of the final version of the instruments

\section{Results}

The instruments were tested on 14 models and 19 cadavers and we used the final version in 2 successful surgeries so far. The greatest result of our work was that the instruments were up to standard in the intraoperative setting (Figure 15).

\section{Discussion}

The Intraosseal Structural Graft (ISG) technique belongs to the group of bulk bone graft techniques used during the replacement of dysplastic hips which presently is under clinical introduction at our Department. The operation can be performed with the usually available orthopaedic surgical instruments, but in order to make the proximally hinged graft preparation easier we decided to develop special instruments. The 3D model testing and cadaver experimentations and numerously modified instrument set proved the success of the developmental work in the intraoperative setting.

\section{REFERENCES}

1. Ikeuchi M, Kawakami T, Kitaoka K, Okanoue Y, Tani $T$. Total hip arthroplasty with a sliding iliac graft for acetabular dysplasia. J Bone Joint Surg Br 2005 May;87(5):635-9.

2. Harris $W H$, Crothers $O, O h I$. Total hip replacement and femoral-head bone-grafting for severe acetabular deficiency in adults. J Bone Joint Surg Am 1977 Sep;59(6):752-9.

\section{Hasegawa Y, Iwata H, Iwase T, Kawamoto K,} Iwasada $S$. Cementless total hip arthroplasty with autologous bone grafting for hip dysplasia. Clin Orthop Relat Res 1996 Mar(324):179-86.
4. Yamaguchi T, Naito M, Asayama I, Shiramizu K. Cementless total hip arthroplasty using an autograft of the femoral head for marked acetabular dysplasia: case series. J Orthop Surg (Hong Kong) 2004 Jun;12(1):14-8.

5. Feugier P, Fessy MH, Bejui J, Bouchet A. Acetabular anatomy and the relationship with pelvic vascular structures. Implications in hip surgery. Surg Radiol Anat 1997;19(2):85-90.

\section{Dr. János Szabó}

Department of Orthopaedic Surgery, Medical and Health Science Center, University of Debrecen H-4032 Debrecen Nagyerdei krt. 98.

Tel.: (+36) 52 255-815 